\title{
Occurrence and antimicrobial resistance of enterococci isolated from goat's milk
}

\author{
Marlena Gołaś-Prądzyńska, Jolanta G. Rola ${ }^{\circledR}$ \\ Department of Hygiene of Food of Animal Origin, \\ National Veterinary Research Institute, 24-100 Puławy, Poland \\ jolanta.rola@piwet.pulawy.pl
}

Received: August 30, 2021

Accepted: December 14, 2021

\begin{abstract}
Introduction: Enterococci are widespread, being part of the bacterial flora of humans and animals. The food chain can be therefore considered as the main route of transmission of antibiotic resistant bacteria between the animal and human populations. Milk in particular represents a source from which resistant bacteria can enter the human food chain. The aim of the study was to determine the occurrence and resistance to antimicrobial agents of Enterococcus spp. strains isolated from raw goat's milk samples. Material and Methods: A total of 207 goat's milk samples were collected. Samples were cultivated on selective media and confirmed as E. faecium or E. faecalis and screened for selected resistance genes by PCR. Drug susceptibility determination was performed by microdilution on Sensititre EU Surveillance Enterococcus EUVENC Antimicrobial Susceptibility Testing (AST) Plates and Sensititre US National Antimicrobial Resistance Monitoring System Gram Positive CMV3AGPF AST Plates. Results: Enterococcal strains totalling 196 were isolated, of which $40.8 \%$ were E. faecalis and $15.3 \%$ were E. faecium. All tested isolates were susceptible to linezolid, penicillin and tigecycline. For most other antimicrobials the prevalence of resistance was $0.5-6.6 \%$ while high prevalence of quinupristin/dalfopristin (51.5\%), tetracycline (30\%) and lincomycin (52\%) resistance was observed. Conclusion: This study affords better knowledge concerning the safety of raw goat's milk in terms of the enterococci possible to isolate from this foodstuff. It seems that enterococci in milk are still mostly susceptible to antimicrobials of major concern as multiply resisted drugs, such as gentamycin and vancomycin. However, the presence of multi-resistant strains in goat milk is cause for apprehension.
\end{abstract}

Keywords: goat's milk, milk, enterococci, antimicrobial resistance, resistance genes.

\section{Introduction}

Enterococci are lactic acid bacteria, an unavoidable presence in the food chain and widespread in human, animal and plant microflora. Commensal on mucosal surfaces and skin, they also inhabit the digestive tracts of many animal species, but their prevalence and distribution vary according to animal species $(14,21)$. They are not only a prevalent species among the dairy microbial community but also well-documented opportunistic pathogens $(26,27)$.

Enterococci are resistant to adverse environmental conditions, including temperature. They can survive manufacturing processes used in food processing, such as fermentation, pasteurisation or cooking. For this reason, enterococci are considered by many authors to be good indicators of the hygiene of the food production process (23). Enterococci play an important role in the production of certain types of ripening cheeses and fermented sausages produced in the Mediterranean region. Through their proteolytic and lipolytic activity, they contribute to the development of the desired organoleptic characteristics $(9,24,26)$. Enterococcal facilitation of cheese and sausage ripening recommends these bacteria as a component of starter cultures. However, the use of enterococci in food processing is sometimes controversial in terms of food safety, mainly because of the possibility of transferring resistance genes $(20,23,27)$. Enterococci can also cause spoilage $(10,25)$. The thermostable amines tyramine, histamine, phenylethylalanine, cadaverine and putrescine which they produce may cause allergic reactions or even food poisoning (23).

An increasing number of bacteria strains have become antimicrobial resistant and some of them have even become multidrug resistant. Enterococci are naturally resistant to low levels of $\beta$-lactams, aminoglycosides and clindamycin, cephalosporins, 
lincosamides, and nalidixic acid (6, 23). They demonstrate a remarkable ability to acquire resistance to antimicrobial agents through exchange of resistanceencoding genes carried on transposons and plasmids. The possibility of plasmid transfer into Staphylococcus aureus, Listeria monocytogenes, Bacillus spp., Clostridium spp. and Lactobacillus spp. has been demonstrated (23). Higher antimicrobial resistance in enterococci may be a consequence of excessive use of antimicrobials in animals. It was found that on farms where antimicrobial agents were used, a significantly higher level of resistant bacteria isolated from animals or humans was reported $(16,19)$. Transfer of resistant bacteria or resistance genes from animals to humans via the food chain is considered possible $(18,29)$. This is the reason why genotypic detection of particular genes causing resistance should complement the determination of phenotypic antimicrobial resistance. When the phenotypic and genotypic resistance patterns are in agreement, the resistance situation is completely apparent; however, a phenotypically resistant bacterium strain may be genotypically susceptible. This is usually because of the omission of appropriate genes from test patterns or the existence of unknown resistance genes (21).

To prevent the transfer of antimicrobial-resistant bacteria from animals to fermented or other foods, the raw substrate (milk or meat) should be pasteurised or heat treated. In addition, the generation of antimicrobial resistant bacteria in food-producing animals and plants has to be minimised by prudent use of antimicrobial agents. To preserve the lifesaving potential of antimicrobials the spread of resistant genes must be stopped at all levels. The ban of antimicrobial drugs with clinical application as growth promoters in animal husbandry is an apt measure to curtail the spread of resistance (21).

The food chain can be considered the main route of transmission of antimicrobial-resistant bacteria between the animal and human populations. As a source of resistant bacteria in the human food chain, milk has particular significance. Moreover, fermented dairy products and fermented meats that are not heat treated before consumption provide a vehicle for antimicrobialresistant bacteria and a direct pathway between the animal intestinal microbiota and the human gastrointestinal tract (21). Provisions of food safety should exclude the possibility of consumer exposure to their virulence through consumption of dairy products (26). Goat's milk is a very good product in terms of both its dietary and pro-health properties. The interest of consumers in goat's milk is growing but data from Poland on its safety are insufficient. It is constructive to investigate whether it is safe in terms of the presence of pathogenic bacteria and their susceptibility to antimicrobials. The aim of the study was to determine the occurrence and resistance to antimicrobial agents of Enterococcus spp. strains isolated from raw goat's milk.

\section{Material and Methods}

Bacterial strains. A total of 207 raw milk samples were collected from goat farms located in Poland during 2019-2020. The samples came from farms located in voivodships in the south and west of Poland. Samples were transported to the Department of Hygiene of Food of Animal Origin in the National Veterinary Research Institute in Poland under refrigeration conditions and analysed on the day of delivery to the laboratory.

Raw milk samples in $1 \mathrm{~mL}$ aliquots were inoculated into $9 \mathrm{~mL}$ of medium supplemented with sodium azide and crystal violet and incubated overnight at $37^{\circ} \mathrm{C}$. Positive samples were inoculated on Slanetz and Bartley medium and incubated at $37^{\circ} \mathrm{C}$ for $24-48 \mathrm{~h}$ until clear growth was obtained. Two to three distinct colonies were chosen for further identification. Pure cultures were stored in Microbank vials (Pro-Lab Diagnostics, Ontario, ON, Canada) at $-70^{\circ} \mathrm{C}$ until analysis.

Isolation of DNA. Total DNA was extracted from the overnight cultures using the Genomic Mini kit (A\&A Biotechnology, Gdańsk, Poland), according to the manufacturer's protocol. The bacterial cell suspension was lysed in a lysis buffer with lysozyme. Proteinase $\mathrm{K}$ was then added to degrade cellular proteins and release genomic DNA from their binding proteins and to eliminate cellular nucleases. Then the sample was transferred to mini columns and bound to the membrane. Purified DNA was eluted with Tris buffer. The samples were stored at $-20^{\circ} \mathrm{C}$ until analysis.

Identification of Enterococcus faecium and Enterococcus faecalis species using PCR. The PCR reaction was based on the EU reference laboratory antimicrobial resistance (EURL-AR) protocol with minor modifications (7). As positive controls, E. faecalis (ATCC 29212) and E. faecium (BM4147) were used, and as the negative control, deionised water was used in place of the template for each test procedure. A TProfessional Basic Gradient 96 thermocycler (Biometra/Analityk Jena, Germany) was used in the amplification process. Electrophoresis of the PCR amplicons was performed in $2 \%$ agarose gel in $1 \times$ Tris/borate/ethylenediaminetetraacetic acid buffer (EURx, Gdańsk, Poland). The gels were visualised under UV light in a GelDoc XR+ System transilluminator (Bio-Rad, Hercules, CA, USA).

Detection of resistance genes. Selected resistance genes were detected in all isolated strains of E. faecalis and $E$. faecium. The presence of genes most commonly involved in resistance to tetracycline was investigated according to Agersø et al. (2) (tet $(L)$ and $\operatorname{tet}(M))$ and de Vries et al. (5) $(\operatorname{tet}(W))$. The detection protocols used E. faecalis - CG 110::Tn916 (tet(M) gene) and E. faecalis - ENT 14.1 (EQAS 2019) (tet $(L)$, tet $(W)$, $v g a A$ genes) as positive controls. The presence of genes involved in resistance to macrolides and streptogramins was investigated by PCRs respectively according to Jensen et al. (16) (ermB) and Hammerum et al. (14) (vgaA and vatD genes). Enterococcus faecalis 
JH2-2::Tn1545 (ermB gene) and E. faecium BM4145 (vatD gene) served as the positive controls. The strains were also tested for the presence of genes responsible for resistance to vancomycin (1) designating $E$. faecium BM4147 for the VanA gene and E. faecalis V583 for the $\operatorname{Van} B$ gene as the positive controls.

Antimicrobial susceptibility testing. Susceptibility to 18 antimicrobials representative of the major classes of critically important antimicrobial agents (Table 2) was tested by broth microdilution using custom Sensititre EU Surveillance Enterococcus EUVENC Antimicrobial Susceptibility Testing (AST) Plates and Sensititre US National Antimicrobial Resistance Monitoring System Gram Positive CMV3AGPF AST Plates (Trek Diagnostics, East Grinstead, UK). As it was for the PCR, E. faecalis ATCC 29212 was used as reference strain for the minimum inhibitory concentration (MIC) method. Briefly, saline suspensions with an optical density (OD) of 0.5 McFarland turbidity standard were produced from overnight cultures on Slanetz and Bartley medium. Next, $30 \mu \mathrm{L}$ of the prepared bacterial suspension was added to $11 \mathrm{~mL}$ of cation-adjusted Mueller-Hinton broth with Tris/ethylenediaminetetraacetic acid/sucrose (CAMHBT) buffer. An aliquot of $50 \mu \mathrm{L}$ of prepared bacterial suspension was transferred to each well on the microplate, and next the microplates were incubated for $18-24 \mathrm{~h}$ at $37 \pm 1^{\circ} \mathrm{C}$. The readings were made using a Sensititre Vizion Digital MIC Viewing System (Trek Diagnostics Systems, Cleveland, OH, USA).

The antimicrobial panels and cut-off values used for the interpretation of the MIC results were in accordance with the guidelines of the European Committee for Antimicrobial Susceptibility Testing (EUCAST), the EURL-AR (8) and the Clinical \& Laboratory Standards Institute document M100 S29 Performance Standard for Antimicrobial Susceptibility Testing, 29th edition (4).

\section{Results}

Positive growth of Enterococcus spp. was obtained from 172 of the 207 collected goat's milk samples $(83.1 \%)$. The number of enterococcal isolates obtained from these samples is shown in Table 1. Of the 196 isolates of enterococci investigated, $80(40.8 \%)$ were identified as E. faecalis, $30(15.3 \%)$ were E. faecium, and 86 (43.9\%) were other Enterococcus species.

Antimicrobial susceptibility results (Table 2) revealed that all isolates were susceptible to linezolid, penicillin and tigecycline. For other substances such as: ampicillin, ciprofloxacin, daptomycin, gentamicin, teicoplanin and vancomycin, kanamycin, streptomycin and nitrofurantoin, chloramphenicol, and erythromycin, the rates of resistance among isolates were in the range of $0.5-6.6 \%$. High prevalences of quinupristin/ dalfopristin (51.5\%), tetracycline (30\%) and lincomycin $(52 \%)$ resistance were observed.
The E. faecalis strains mainly showed resistance to tetracycline $(62.5 \%)$ but were also resistant to erythromycin $(10 \%)$ and chloramphenicol $(5 \%)$. The E. faecium isolates most commonly exhibited resistance to nitrofurantoin (30\%), erythromycin $(20 \%)$ and tetracycline (10\%).

The prevalence of multi-drug resistant strains was also compared (Table 3). Resistance by E. faecalis to two $(35 \%)$ or three $(40 \%)$ substances was the most common, but strains resistant to more antimicrobials were also observed. Among E. faecium isolates, resistance to one (33.3\%) or two (36.7\%) antimicrobials was the most common. Resistance to more than four substances concurrently was not observed among E. faecium isolates.

Most enterococci (69.4\%) were resistant to one or more of the drugs tested, while $30.6 \%$ of isolates had no resistance. Of those $69.4 \%, 50 \%$ were multi-resistant (resistant to two or more antimicrobials), and the other $19.4 \%$ were single antimicrobial resistant. The frequency of antimicrobial resistance in these strains is shown in Table 3, as is the size of the subgroup of antimicrobials to which they had resistance, which ranged from one to eight. Most (21.9\%) showed resistance to two drugs, $19.4 \%$ showed resistance to one antimicrobial, $17.9 \%$ to three, $2.0 \%$ to four, and $5.6 \%$ to five. Some strains of E. faecalis showed resistance to eight antimicrobials, in contrast to those of E. faecium, which not one showed resistance to more than four substances.

Enterococcus faecalis and E. faecium isolates were analysed for the presence of selected antimicrobial resistance genes (Table 4). Strains were analysed for resistance to vancomycin (detection of the VanA and VanB genes), macrolides, lincosamides, streptogramins $\mathrm{B}$ (detection of the ermB gene), tetracyclines (detection of the $\operatorname{tet}(L), \operatorname{tet}(M)$ and $\operatorname{tet}(W)$ genes) and streptogramins (detection of the vgaA and vatD genes). The $\operatorname{Van} A, \operatorname{Van} B, \operatorname{tet}(L), \operatorname{tet}(W)$ and $\operatorname{vat} D$ genes were not detected among the analysed isolates. The ermB gene was detected in the genetic material of four E. faecalis isolates which were phenotypically resistant to erythromycin. The $\operatorname{tet}(M)$ gene was detected in the genetic material of 43 tetracycline-resistant $E$. faecalis isolates. Sixteen isolates harboured the vgaA gene (15 E. faecalis and one E. faecium). All E. faecalis strains showed phenotypic streptogramin resistance.

Table 1. Species of enterococci isolated from raw goat's milk samples

\begin{tabular}{lc}
\hline Species & $\begin{array}{c}\text { Number of raw goat milk } \\
\text { samples }\end{array}$ \\
\hline E. faecalis $(\mathrm{n}=80)$ & 62 \\
E. faecium $(\mathrm{n}=30)$ & 23 \\
Enterococcus spp. $(\mathrm{n}=86)$ & 67 \\
E. faecalis + E. faecium & 5 \\
E. faecalis + Enterococcus spp. & 13 \\
E. faecium + Enterococcus spp. & 2 \\
\hline Total & 172 \\
\hline
\end{tabular}


Table 2. Prevalence of enterococci resistant to antimicrobial agents isolated from raw goat's milk samples

\begin{tabular}{lcccc}
\hline \multirow{2}{*}{ Antimicrobial agent } & \multicolumn{4}{c}{ Number $(\%)$ of resistant strains } \\
\cline { 2 - 5 } Ampicillin & $1(1.25)$ & $1(3.3)$ & $0(0)$ & $2(1)$ \\
Chloramphenicol & $4(5)$ & $0(0)$ & $0(0)$ & $4(2)$ \\
Ciprofloxacin & $1(1.25)$ & $0(0)$ & $0(0)$ & $1(0.5)$ \\
Daptomycin & $0(0)$ & $0(0)$ & $4(4.65)$ & $4(2)$ \\
Erythromycin & $8(10)$ & $6(20)$ & $0(0)$ & $14(7)$ \\
Gentamicin & $1(1.25)$ & $0(0)$ & $0(0)$ & $1(0.5)$ \\
Kanamycin & $12(15)$ & $1(3.3)$ & $0(0)$ & $13(6.6)$ \\
Lincomycin & $79(98.75)$ & $22(73.3)$ & $1(1.16)$ & $102(52)$ \\
Linezolid & $0(0)$ & $0(0)$ & $0(0)$ & $0(0)$ \\
Nitrofurantoin & $2(2.5)$ & $9(30)$ & $0(0)$ & $11(5.6)$ \\
Penicillin & $0(0)$ & $0(0)$ & $0(0)$ & $0(0)$ \\
Streptomycin & $11(13.75)$ & $0(0)$ & $0(0)$ & $11(5.6)$ \\
Teicoplanin & $1(1.25)$ & $0(0)$ & $2(2.33)$ & $3(1.5)$ \\
Tetracycline & $50(62.5)$ & $3(10)$ & $6(6.97)$ & $59(30)$ \\
Tigecycline & $0(0)$ & $0(0)$ & $0(0)$ & $0(0)$ \\
Tylosin & $7(8.75)$ & $1(3.3)$ & $0(0)$ & $8(4.1)$ \\
Quinupristin/Dalfopristin & $80(100)$ & $2(6.6)$ & $19(22.09)$ & $101(51.5)$ \\
Vancomycin & $1(1.25)$ & $0(0)$ & $3(3.48)$ & $4(2)$ \\
\hline
\end{tabular}

Table 3. Frequency distribution of the resistance patterns of enterococci isolated from raw goat's milk samples to antimicrobial agents

\begin{tabular}{lcccc}
\hline \multirow{2}{*}{ Antimicrobial resistance pattern } & \multicolumn{4}{c}{ Number $(\%)$ of isolates with antimicrobial resistance patterns } \\
& Total $(\mathrm{n}=196)$ & E. faecalis $(\mathrm{n}=80)$ & E. faecium $(\mathrm{n}=30)$ & Enterococcus spp. $(\mathrm{n}=86)$ \\
\hline No resistance & $60(30.6)$ & $0(0)$ & $5(16.7)$ & $55(64)$ \\
Single resistance & $38(19.4)$ & $1(1.3)$ & $10(33.3)$ & $27(31.4)$ \\
Multiple resistance & $98(50)$ & $79(98.7)$ & $15(50)$ & $4(4.6)$ \\
2 antimicrobials & $43(21.9)$ & $28(35)$ & $11(36.7)$ & $4(4.6)$ \\
3 antimicrobials & $35(17.9)$ & $32(40)$ & $3(10)$ & - \\
4 antimicrobials & $4(2)$ & $3(3.75)$ & $1(3.3)$ & - \\
5 antimicrobials & $11(5.6)$ & $11(13.75)$ & - & - \\
7 antimicrobials & $2(1)$ & $2(2.5)$ & - & - \\
8 antimicrobials & $3(1.5)$ & $3(3.7)$ & - & - \\
\hline
\end{tabular}

Table 4. Antimicrobial agent resistance determinants of the Enterococcus faecalis and Enterococcus faecium isolates

\begin{tabular}{|c|c|c|c|c|}
\hline Species & \multicolumn{3}{|c|}{ Antimicrobial resistance gene content } & $\begin{array}{c}\text { Number } \\
\text { of R strains }\end{array}$ \\
\hline \multirow{11}{*}{ E. faecalis $(\mathrm{n}=80)$} & \multirow{3}{*}{ Glycopeptides } & $\operatorname{Van} A$ & 0 & \multirow{3}{*}{1} \\
\hline & & $\operatorname{Van} B$ & 0 & \\
\hline & & NP & 80 & \\
\hline & \multirow{4}{*}{ Tetracyclines } & $\operatorname{tet}(L)$ & 0 & \multirow{4}{*}{50} \\
\hline & & $\operatorname{tet}(M)$ & 43 & \\
\hline & & $\operatorname{tet}(W)$ & 0 & \\
\hline & & $\mathrm{NP}$ & 37 & \\
\hline & \multirow{4}{*}{$\begin{array}{l}\text { Macrolides, } \\
\text { lincosamides, } \\
\text { streptogramins }\end{array}$} & ermB & 4 & \multirow{4}{*}{79} \\
\hline & & vatD & 0 & \\
\hline & & $v g a A$ & 15 & \\
\hline & & NP & 62 & \\
\hline \multirow{11}{*}{ E. faecium $(\mathrm{n}=30)$} & \multirow{3}{*}{ Glycopeptides } & $\operatorname{Van} A$ & 0 & \multirow{3}{*}{0} \\
\hline & & $\operatorname{Van} B$ & 0 & \\
\hline & & NP & 30 & \\
\hline & \multirow{4}{*}{ Tetracyclines } & $\operatorname{tet}(L)$ & 0 & \multirow{4}{*}{3} \\
\hline & & $\operatorname{tet}(M)$ & 0 & \\
\hline & & $\operatorname{tet}(W)$ & 0 & \\
\hline & & NP & 30 & \\
\hline & \multirow{4}{*}{$\begin{array}{l}\text { Macrolides, } \\
\text { lincosamides, } \\
\text { streptogramins }\end{array}$} & ermB & 1 & \multirow{4}{*}{22} \\
\hline & & vatD & 0 & \\
\hline & & $\operatorname{vgaA}$ & 1 & \\
\hline & & NP & 28 & \\
\hline
\end{tabular}

$\mathrm{R}$ - resistant strains; NP - not present 


\section{Discussion}

Epidemiological data show that enterococci are among the most important nosocomial pathogens. Monitoring is in place only for infections by E. faecium and E. faecalis as the main species most frequently invading animal and human organisms (27). Enterococci, in particular E. faecium and E. faecalis, are present in goat's milk as part of the natural microflora and because of the various genetic transfer mechanisms are a potential source of resistance that can be transferred to human strains $(12,13)$. Our study isolated enterococci from a high percentage of goat's milk samples (83.1\%). In the paper of Výrostková et al. (30), the majority of Enterococcus strains from ruminant milk cheese samples were identified as E. faecium (76\%) or E. faecalis $(23 \%)$. Similarly to other authors, we identified high percentages of E. faecalis (40.8\%) and E. faecium $(15.3 \%)$ strains in the goat milk tested in the present research $(12,13,22,30)$.

The easy acquisition of antimicrobial resistance by enterococci likely contributes to their emergence as major nosocomial pathogens and therefore the question arises of how to manage foodborne enterococci. Despite the use of molecular typing, it is still difficult to distinguish between foodborne and clinical isolates (20). Intrinsic enterococcal resistance against cephalosporins, clindamycin, trimethoprimsulfamethoxazole, fluoroquinolones, low concentrations of aminoglycosides, and $\beta$-lactams has been reported. In addition, strains can develop resistance to tetracycline, chloramphenicol, rifampin, glycopeptides, quinolones, macrolides, lincosamides, streptogramins, high levels of aminoglycosides, and $\beta$-lactams by mutation or acquisition of genetic material carried by transposons or plasmids (3). Gaglio et al. (12) observed a high level of resistance to antibacterial agents in enterococci isolated from cheese. As did other authors, we observed enterococci resistant to drugs used to treat human infections and the most prevalent E. faecalis and E. faecium were resistant to two or more of them. From this perspective, there is a need to expand the knowledge of Enterococcus strains present in raw goat's milk and dairy products. Precise research is necessary to confirm the safety of their adoption in the diet in regard to the risk of enterococcitis in humans; however, so far, no case of nosocomial infection has been confirmed after consuming dairy products.

Since penicillins, glycopeptides, and aminoglycosides are the most common therapeutic options for the treatment of enterococcal infections, it was a role in the spread of resistance to these agents which our study highlighted for the strains isolated from raw goat's milk in Poland. Franz et al. (11) showed resistance of enterococci to the most commonly used antimicrobials. Our results showed single ampicillin-, daptomycin-, gentamycin-, teicoplanin- and vancomycin-resistant Enterococcus strains (VRE). Enterococci have low-level intrinsic resistance to the aminoglycosides because of the limited ability of these agents to penetrate the cell wall (11). We did not observe strains resistant to high-level aminoglycosides, with the exception of some E. faecalis isolates resistant to high-level streptomycin. Contrary to our results, in the study by ChajęckaWierzchowska et al. (3), most of the analysed strains were resistant to aminoglycosides.

Due to the rapid spread of multi-drug resistant strains in the treatment of infections caused by resistant enterococci, the drugs of last resort - linezolid, tigecycline and daptomycin - are of great importance (27). Referring to earlier studies, the authors observed the increasing prevalence of linezolid resistance in E. faecium $(12,30)$. Our study did not reveal strains resistant to linezolid as well as penicillin and tigecycline. Gaglio et al. (12) did not observe strains resistant to vancomycin and linezolid. Similarly, in our study we noticed only a single strain resistant to vancomycin. We observed similar percentages of strains resistant to streptomycin and chloramphenicol, a higher number of strains resistant to tetracycline, and fewer strains resistant to ciprofloxacin than in the cited study (12).

In human medicine, glycopeptides play an important role in the treatment of severe infections caused by staphylococci and streptococci resistant to beta-lactam antibiotics. The widespread use of vancomycin in hospital treatment in the 1990s (USA) and from the year 2000 (Europe) and use of avoparcin (a drug with a similar structure to vancomycin) as an animal feed additive led to the spread of VRE strains (27). No observation was made by Výrostková et al. (30) of vancomycin-resistant strains. In the work of other authors, single vancomycin-resistant strains isolated from ready-to-eat dairy products were observed, but this did not correlate with the presence of the $\operatorname{Van} A$ and $\operatorname{Van} B$ genes, which was also in accordance with our results (3). Výrostková et al. (30), observed a higher level of resistance in E. faecium than in E. faecalis isolates from food and clinical samples. In contrast, we observed the opposite trend - more than half of the E. faecalis strains (62.5\%) and only $10 \%$ of the E. faecium strains showed resistance to tetracycline.

In our study, ciprofloxacin and nitrofurantoin were active against enterococci. These results are encouraging since ciprofloxacin is one of the fluoroquinolones, which usually exhibit weak activity against enterococci, whereas nitrofurantoin is commonly used in the treatment of urinary infections caused by VRE.

The ineffectiveness of the streptogramin combination quinupristin-dalfopristin against E. faecalis was previously described, and this was confirmed in our study. All E. faecalis strains were resistant to this antimicrobial agent (3).

Investigation of the presence of resistance genes in our strains showed that tet(M) was the most frequently detected of them. Tetracycline resistance is often discovered in multidrug-resistant microorganisms and involves different mechanisms, such as efflux pump proteins $($ tet $(K)$ and tet $(L))$ or ribosomal protection 
proteins $(\operatorname{tet}(M), \operatorname{tet}(O)$, and $\operatorname{tet}(S))$. These results are similar to those of Chajecka-Wierzchowska et al. (3) from analysis of enterococci strains isolated from dairy products. Seven strains resistant to tetracycline carried the tet $(M)$ but not the tet $(K)$ gene. Almost all E. faecalis strain $(86 \%)$ resistant to tetracycline carried the $\operatorname{tet}(M)$ but not the tet $(L)$ or $\operatorname{tet}(W)$ genes. The tet $(M)$ gene was found in all 43 E. faecalis strains positive for phenotypic resistance. Seven phenotypically resistant strains did not possess any of the tested tetracycline resistance genes $(13,26)$.

The ermB gene is the most prevalent macrolide resistance gene among enterococci. In the study by Gaglio et al. (12), the ermB gene was not detected among the tested resistant strains. In contrast, we observed five E. faecalis strains containing the ermB gene but only two were also phenotypically resistant to erythromycin, suggesting the existence of a silent gene. It may be that gene expression also depends on environmental and culture conditions. This lack of correlation between the resistance exhibited by phenotypes and the resistance apparent for genotypes highlights the need for the two corresponding analytical approaches to be used jointly to investigate enterococcal strains as foodborne hazards $(13,26)$. Due to the risk of the presence of strains resistant to glycopeptides, the authors tested enterococci for the presence of the relevant resistance genes (VanA and $\operatorname{VanB}$ ). The low incidence of VRE strains noticed in this study is in agreement with the findings of other researchers, who reported low or no incidence of vancomycin resistance in European cheeses, suggesting that this acquired resistance is still restricted to the clinical setting (26). In our study, we observed one E. faecalis strain resistant to vancomycin. None of the tested strains had the VanA and VanB genes. In conclusion, the isolates in our study showed resistance to at least one tested antimicrobial agent. The widespread use of antimicrobial agents in the treatment of mastitis, in particular, exerts selection pressure and thus generates resistance among enterococci isolated from milk $(12,22)$.

This study expands knowledge of the pharmacotherapeutic treatability aspects of enterococci isolated from raw goat milk. Enterococci in milk are still mostly susceptible to antimicrobials of major concern as multiply resisted drugs, such as gentamycin, vancomycin, linezolid and tigecycline. However, the presence of multi-resistant strains in goat's milk is disturbing. Dairy products constitute a reservoir of enterococci and a potential reservoir of antimicrobial resistance and virulence genes which could be transferred to human strains. Enterococci can be a source of resistance genes for other bacteria, including Gram-negatives such as Campylobacter and Salmonella. Moreover, the evaluation of enterococci safety through in vitro expression of virulence traits does not always reflect the real hazard in these microorganisms because of the presence of silent genes, which could potentially be activated by particular environmental conditions, thus transforming these bacteria into pathogens or enhancing their pathogenicity (26).

In the future, research should focus on carefully examining the potential for antimicrobial resistance to be transmitted between strains isolated from food and human enterococci. Greater effort should be directed to reducing the use of antimicrobials in veterinary and human medicine, which may eliminate resistant enterococci from food (20). The use of antimicrobial agents in veterinary medicine and as feed additives should be reduced to a minimum, and only when required and when all alternatives are excluded. Besides increased care to maintain or improve hygienic conditions, these are the necessary conditions for health and environmental protection against the risk of harm to human health from these bacteria (28).

Conflict of Interests Statement: The authors declare that there is no conflict of interests regarding the publication of this article.

Financial Disclosure Statement: This study was funded by the statutory activity of the National Veterinary Research Institute in Puławy.

Animal Rights Statement: Not applicable.

\section{References}

1. Aarestrup F.M.: Glycopeptide susceptibility among Danish Enterococcus faecium and Enterococcus faecalis isolates of animal and human origin and PCR identification of genes within the vana cluster. Antimicrob Agents Chemother 1996, 40, 19381940, doi: 10.1128/AAC.40.8.1938.

2. Agersø Y., Jensen L.B., Givskov M., Roberts M.C.: The identification of a tetracycline resistance gene tet(M), on a Tn916like transposon, in the Bacillus cereus group. FEMS Microbiol Lett 2002, 214, 251-256, doi: 10.1111/j.1574-6968.2002. tb11355.x.

3. Chajęcka-Wierzchowska W., Zadernowska A., García-Solache M. Ready-to-eat dairy products as a source of multidrug-resistant Enterococcus strains: Phenotypic and genotypic characteristics. J Dairy Sci 2020, 103, 4068-4077, doi: 10.3168/jds.2019-17395.

4. Clinical and Laboratory Standards Institute: M100-S29 Performance Standards for Antimicrobial Susceptibility Testing; Twenty ninth Informational Supplement. CLSI, Wayne, 2019.

5. de Vries L.E., Vallès Y., Agersø Y., Vaishampayan P.A., García-Montaner A., Kuehl J.V., Christensen H., Barlow M., Francinoet P.M.: The Gut as Reservoir of Antibiotic Resistance: Microbial Diversity of Tetracycline Resistance in Mother and Infant. PLoS One 2011, 6, e21644, doi: 10.1371/journal.pone.0021644.

6. Drahovska H., Slobodnikova L., Kocincova D., Seman M., Končekova R., Trupl J., Turňa J.: Antibiotic resistance and virulence factors among clinical and food enterococci isolated in Slovakia. Folia Microbiol 2004, 49, 763-768, doi: 10.1007/BF02931562.

7. Dutka-Malen S., Evers S., Courvalin P.: Detection of glycopeptide resistance genotypes and identification to the species level of clinically relevant enterococci by PCR. J Clin Microbiol 1995, 33 , 24-27, doi: 10.1128/jcm.33.1.24-27.1995.

8. European Committee on Antimicrobial Susceptibility Testing: Breakpoint tables for interpretation of MICs and zone diameters, Version 9.0. EUCAST, Växjö, 2019. https://www.eucast. org/ast_of_bacteria/previous_versions_of_documents/. 
9. Foulquie Moreno M.R., Sarantinopoulos P., Tsakalidou E., De Vuyst L.: The role and application of enterococci in food and health. Int J Food Microbiol 2006, 106, 1-24, doi: 10.1016/j.ijfoodmicro.2005.06.026.

10. Franz C.M.A.P., Huch M., Abriouel H., Holzapfel W., Gálvez A.: Enterococci as probiotics and their implication in food safety. Int J Food Microbiol 2011, 151, 125-140, doi: 10.1016/j.ijfoodmicro.2011.08.014.

11. Franz C.M.A.P., Muscholl-Silberhorn A.B., Yousif N.M.K., Vancanneyt M., Swings J., Holzapfel W.H.: Incidence of virulence factors and antibiotic resistance among enterococci isolated from food. Appl Environ Microbiol 2001, 67, 4385-4389, doi: 10.1128/AEM.67.9.4385-4389.2001.

12. Gaglio R., Couto N., Marques C., de Fatima Silva Lopes M., Moschetti G., Pomba C., Settanni L.: Evaluation of antimicrobial resistance and virulence of enterococci from equipment surfaces, raw materials, and traditional cheeses. Int J Food Microbiol 2016, 236, 107-14, doi: 10.1016/j.ijfoodmicro.2016.07.020.

13. Garrido A.M., Gálvez A., Pulido R.P.: Antimicrobial Resistance in Enterococci. J Infect Dis Ther 2014, 2, 150, doi: 10.4172/23320877.1000150

14. Giraffa G.: Enterococci from foods. FEMS Microbiol Rev 2002, 26, 163-171, doi: 10.1111/j.1574-6976.2002.tb00608.x.

15. Hammerum A.M., Jensen L.B., Aarestrup F.M.: Detection of the satA gene and transferability of virginiamycin resistance in Enterococcus faecium from food-animals. FEMS Microbiol Lett 1998, 168, 145-151, doi: 10.1111/j.1574-6968.1998.tb13267.x.

16. Hershberger E., Oprea S.F., Donabedian S.M., Perri M., Bozigar P., Barttlett P., Zervos M.J.: Epidemiology of antimicrobial resistance in enterococci of animal origin. $\mathrm{J}$ Antimicrob Chemother 2005, 55, 127-130, doi: 10.1093/jac/dkh508.

17. Jensen L.B., Frimodt-Moeller N., Aarestrup F.M.: Presence of erm gene classes in Gram-positive bacteria of animal and human origin in Denmark. FEMS Microbiol Lett 1999, 170, 151-158, doi: 10.1111/j.1574-6968.1999.tb13368.x.

18. Larsen J., Schonheyder H.C., Lester C.H., Olsen S.S., Porsbo L.J., Garcia-Migura L., Jensen L.B., Bisgaard M., Hammerum A.M.: Porcine-origin gentamicin-resistant Enterococcus faecalis in humans, Denmark. Emerg Infect Dis 2010, 16, 682-684, doi: 10.3201/eid1604.090500.

19. Lukášová J., Šustáčkova A.: Enterococci and antibiotic resistance. Acta Vet Brno 2003, 72, 315-323, doi: 10.2754/ avb200372020315
20. Ogier J.C., Serror P.: Safety assessment of dairy microorganisms: the Enterococcus genus. Int J Food Microbiol 2008, 126, 291-301, doi: 10.1016/j.ijfoodmicro.2007.08.017.

21. Patel A.R., Shah N.P., Prajapati J.B.: Antibiotic Resistance Profile of Lactic Acid Bacteria and Their Implications in Food Chain. World J Dairy Food Sci 2012, 7, 202-211, doi: 10.5829/idosi.wjdfs.2012.7.2.1113.

22. Różańska H., Lewtak-Piłat A., Kubajka M., Weiner M.: Occurrence of Enterococci in Mastitic Cow's Milk and their Antimicrobial Resistance. J Vet Res 2019, 63, 93-97, doi: 10.2478/jvetres-2019-0014.

23. Różańska H., Lewtak-Piłat A., Osek J.: Enterococci multifaceted microorganisms (in Polish). Życie Weterynaryjne 2013, 88, 562-564

24. Ruiz P., Pérez-Martín F., Seseña S., Llanos Palop M.: Seasonal diversity and safety evaluation of enterococci population from goat milk in a farm. Dairy Sci Technol 2016, 96, 359-375, doi: 10.1007/s13594-015-0273-y.

25. Silva N., Igrejas G., Gonçalves A., Poeta P.: Commensal gut bacteria: distribution of Enterococcus species and prevalence of Escherichia coli phylogenetic groups in animals and humans in Portugal. Ann Microbiol 2012, 62, 449-459, doi: 10.1007/s13213-011-0308-4.

26. Silvetti T., Morandi S., Brasca M.: Does Enterococcus faecalis from traditional raw milk cheeses serve as a reservoir of antibiotic resistance and pathogenic traits? Foodborne Pathog Dis 2019, 16, 359-367, doi: 10.1089/fpd.2018.2542.

27. Talaga-Ćwiertnia K., Bulanda M.: Drug resistance in the genus Enterococcus - current problem in humans and animals. Post Mikrobiol 2018, 57, 244-250, doi: 10.21307/PM-2018.57.3.244.

28. Tansuphasiri U., Khaminthakul D., Pandii W.: Antibiotic resistance of enterococci isolated from frozen foods and environmental water. Southeast Asian J Trop Med Public Health 2006, 37, 162-170.

29. Vignaroli C., Zandri G., Aquilanti L., Pasquaroli S., Biavasco F.: Multidrug-resistant enterococci in animal meat and faeces and co-transfer of resistance from Enterococcus durans to a human Enterococcus faecium. Curr Microbiol 2011, 62, 1438-1447, doi: 10.1007/s00284-011-9880-x.

30. Výrostková J., Regecová I., Dudriková E., Mal’ová J., Zigo F., Kováčová M., Illek J.: Antimicrobial resistance of bacterial isolates from sheep and goat cheeses in eastern Slovakia. Pol J Vet Sci 2021, 24, 13-22, doi: 10.24425/pjvs.2020.135814. 